\title{
A Case Report of Skip Lesions in Perianal Mucinous Adenocarcinoma
}

\author{
Nirali Shah, Michael Gates, Dr. Imran Saeed. \\ *Corresponding Author: Nirali Shah. \\ Received date: March 11, 2021; Accepted date: April 12, 2021; Published date: April 30, 2021 \\ Citation: Shah N, Gates M, Saeed I. (2021) A Case Report of Skip Lesions in Perianal Mucinous Adenocarcinoma. J. Surgi Case Repo and Ima 4(3); \\ DOI:10.31579/2690-1897/064
}

Copyright: (C) 2021 Nirali Shah, This is an open access article distributed under the Creative Commons Attribution License, which permits unrestricted use, distribution, and reproduction in any medium, provided the original work is properly cited.

\begin{abstract}
:
Perianal Mucinous Adenocarcinoma (PMA) is a rare gastrointestinal malignancy, known to occur in patients with a long-standing history of recurrent perirectal abscesses or a chronic fistula. In this case report, we present a unique presentation of perianal mucinous adenocarcinoma, which began as a rectal abscess and progressed to a case of perianal mucinous adenocarcinoma, with a skip lesion present all the way up in the mid-rectal region. To the best of our knowledge, this is the first case in which PMA presented with skip lesions as opposed to a continuous lesion, and presented without a long standing history of fistulas and recurrent abscesses, but instead just a 4-day history of perianal burning, itching and tingling. The use of CT imaging, MRI's and PET scans as diagnostic modalities for PMA are further elucidated in the report, along with a discussion of NCCN guidelines on using radiation therapy followed by an APR with inguinal node dissection for the management and treatment of PMA. Sharing this unique and atypical presentation of PMA with multimodality speciality groups and tumor boards helps in the development of various diagnostic and therapeutic approaches for PMA, as well as enhances our understanding of this rare malignant entity.
\end{abstract}

Keywords: perianal mucinous adenocarcinoma, skip lesions, recurrent perirectal abscesses, chronic fistulas

\section{Introduction:}

Perianal mucinous adenocarcinoma (PMA) is a rare, malignant entity constituting $2-3 \%$ of all gastrointestinal malignancies ${ }^{1}$. It is thought to occur in patients with a long-standing history of recurrent perirectal abscesses or a chronic fistula ${ }^{2}$. Presence of an abscess or fistula is speculated to cause repeated friction, obstruction and irritation in the rectal region, thereby triggering an inflammatory response and scarring. The repeated bouts of inflammatory responses render the epithelial cells in that region susceptible to transforming from benign, to dysplastic and eventually to malignant. The anal canal epithelium is columnar in the upper $2 / 3^{\text {rd }}$ and squamous in the lower $1 / 3^{\text {rd }}$. The columnar cells have been arranged to form Columns of Morgagni whose bases have crypts connected to glands which are then connected to ducts. It is the chronic obstruction of these ducts which cause the formation of fistulas and abscesses ${ }^{3}$.

Anal canal tumors are $75 \%$ of the squamous cell type and $25 \%$ of the adenocarcinoma type. Typically, patients with PMA tend to present with perianal pain and itchiness, bleeding per rectum and in most cases an ulcerative growth or a palpable mass with a bloody or mucoid discharge ${ }^{2}$. Most of these cases tend to be preceded by a symptomatic period of 4-6 months, and the patient population to commonly present with PMA includes those with a past medical history of HIV, IBD or recurrent and long-standing fistulas and abscesses ${ }^{4}$. The purpose of this case report is to present a silent and unique case of perianal mucinous adenocarcinoma, which to the best our knowledge is the only case reported in the literature so far with an additional component of mucinous adenocarcinoma skip lesions present higher up in the colon as well.

\section{Case Report:}

PB, a 61-yr old Caucasian male, presented with a 4-day history of mild (3/10 intensity) and continuous rectal pain and a burning sensation upon sitting due to a rectal abscess. The patient had no past medical history of HIV, IBD or recurrent anal fistulas and abscesses, and al so no past social history of alcohol consumption or tobacco usage. The rectal examination revealed a 1-cm soft tissue growth and a CTAP performed at that time revealed a perianal abscess measuring $3.3 * 3.1 * 4.9 \mathrm{~cm}$ with infiltration into the surrounding planes. Purulent material came out upon performing an I and $\mathrm{D}$, and then the patient was discharged with PO antibiotics.

Upon follow-up with the colorectal surgeon 5-days later, another rectal exam revealed a 2-cm soft tissue growth in the form of a mildly tender and fluctuant mass, located in the right perirectal quadrant, close to the anal verge. Visually, it appeared to be a warty lesion and upon pressing the lesion, a gelatinous mucinous material was found to exude out. Subsequently, a lesion excision was performed in the OR, and gelatinous material underneath the lesion was appreciated. There was fluctuance and erythema adjacent to the lesion, but no fissure or anal canal lesion was palpated upon digital canal 
examination. The tumor was $2 \mathrm{~cm}$ in diameter, with foci suspicious vascular invasion. Furthermore, the surgical resection margins were both deep and laterally (+) for tumor. Overlying benign squamous mucosa was found and immunohistochemistry showed (+) CK20, (+) Cdx2 and (-) Ck7; the classic IHC staining pattern for colonic adenocarcinoma.

A.
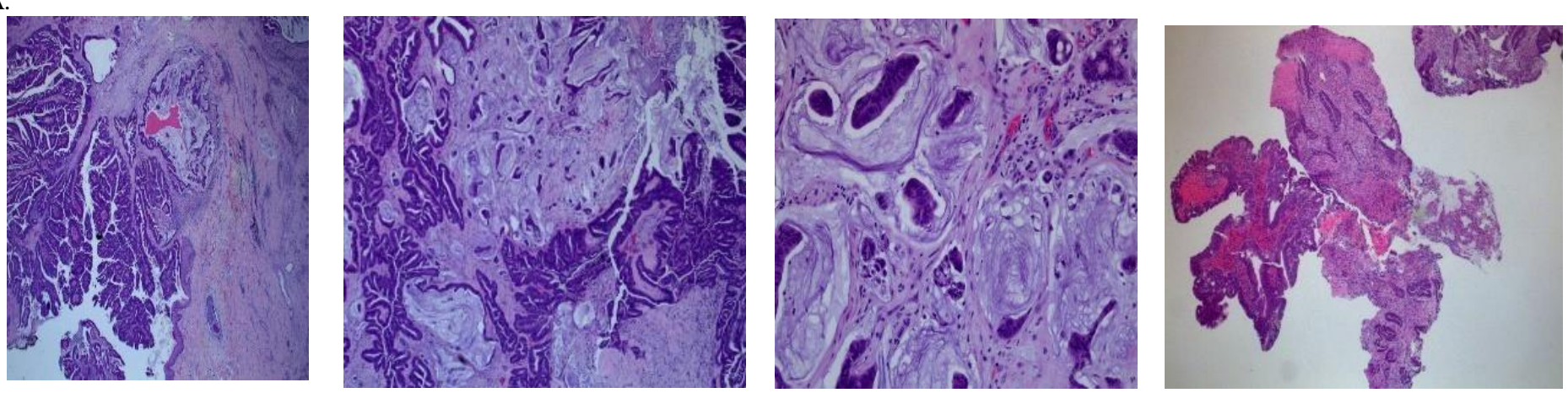

Fig (A): H\&E staining of the first rectal lesion, (+) for moderately differentiated adenocarcinoma

B.

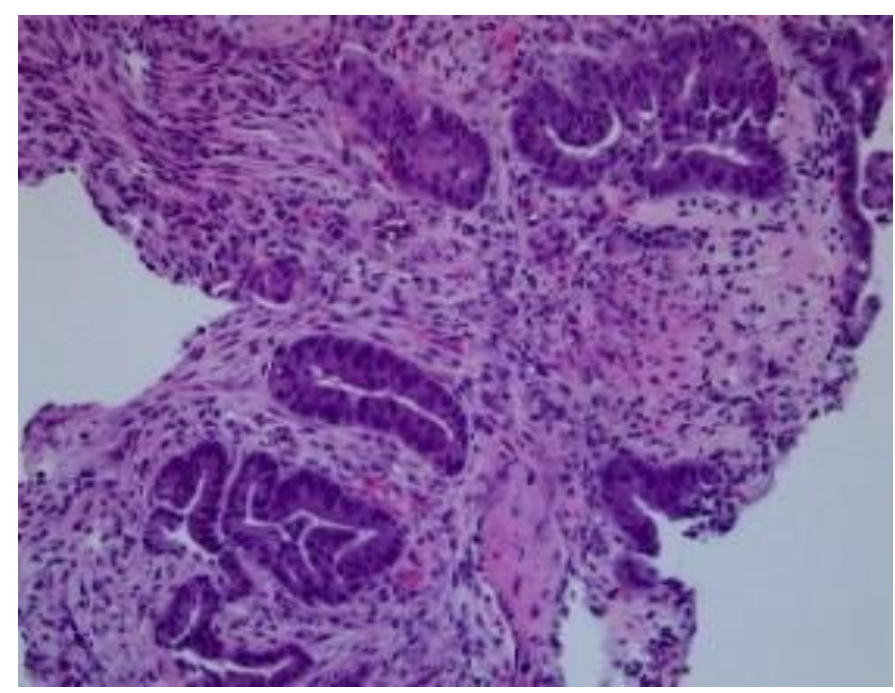

Fig (B): H\&E staining of the mid-rectal mass found upon colonoscopy a few weeks later, also (+) for moderately differeniated adenocarcinoma C.

(a)

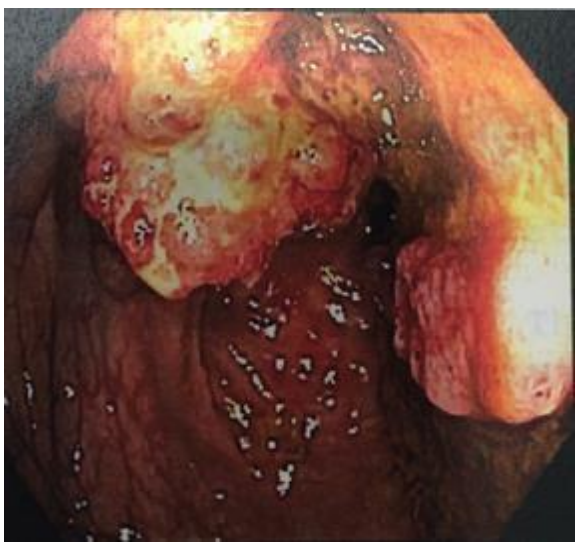

(b)

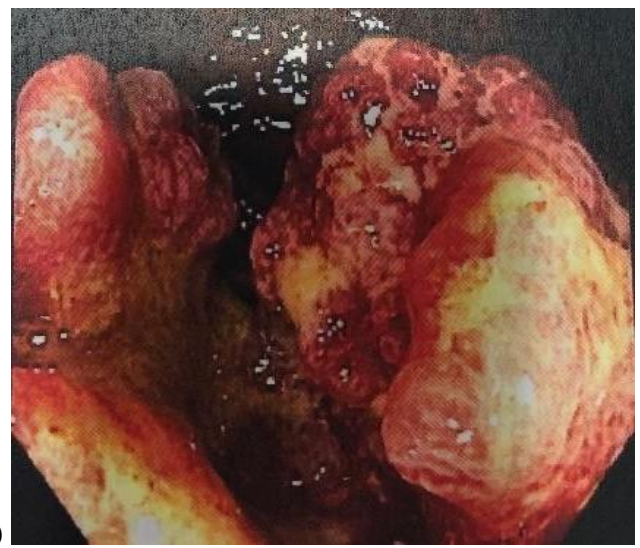

C. (a) (b) Large rectal lesion found in the mid-rectal region upon peforming colonoscopy. Tumor was biopsied and a tattoo was placed distal to the lesion.

D. 
(a)

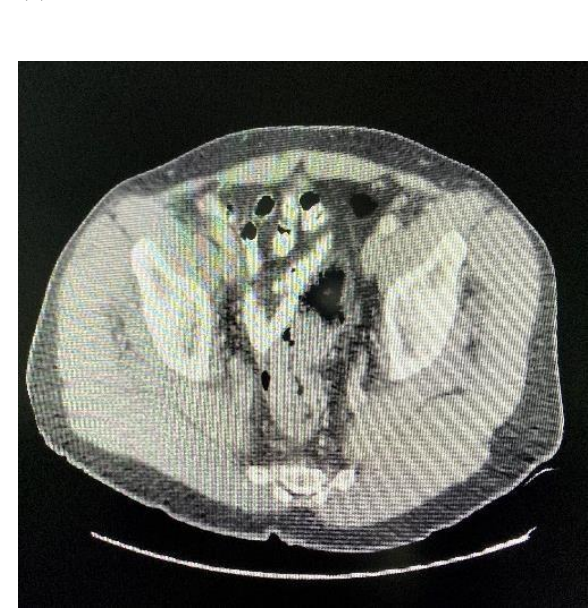

(b)
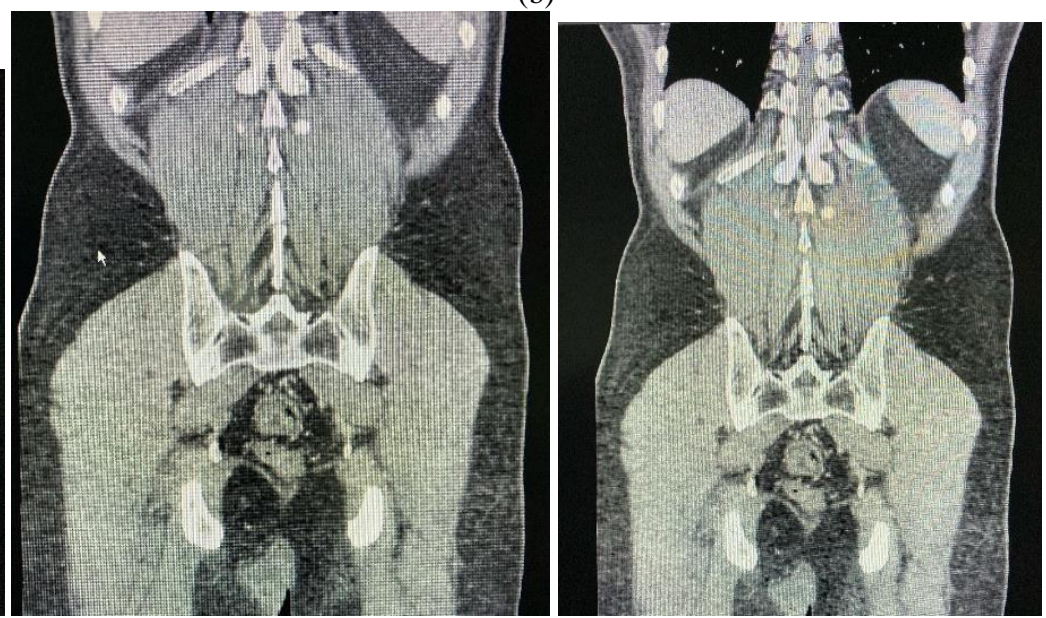

D. (a) CT scan of the rectal lesion. (b) Fluid collection in the perirectal area is appreciated

E.
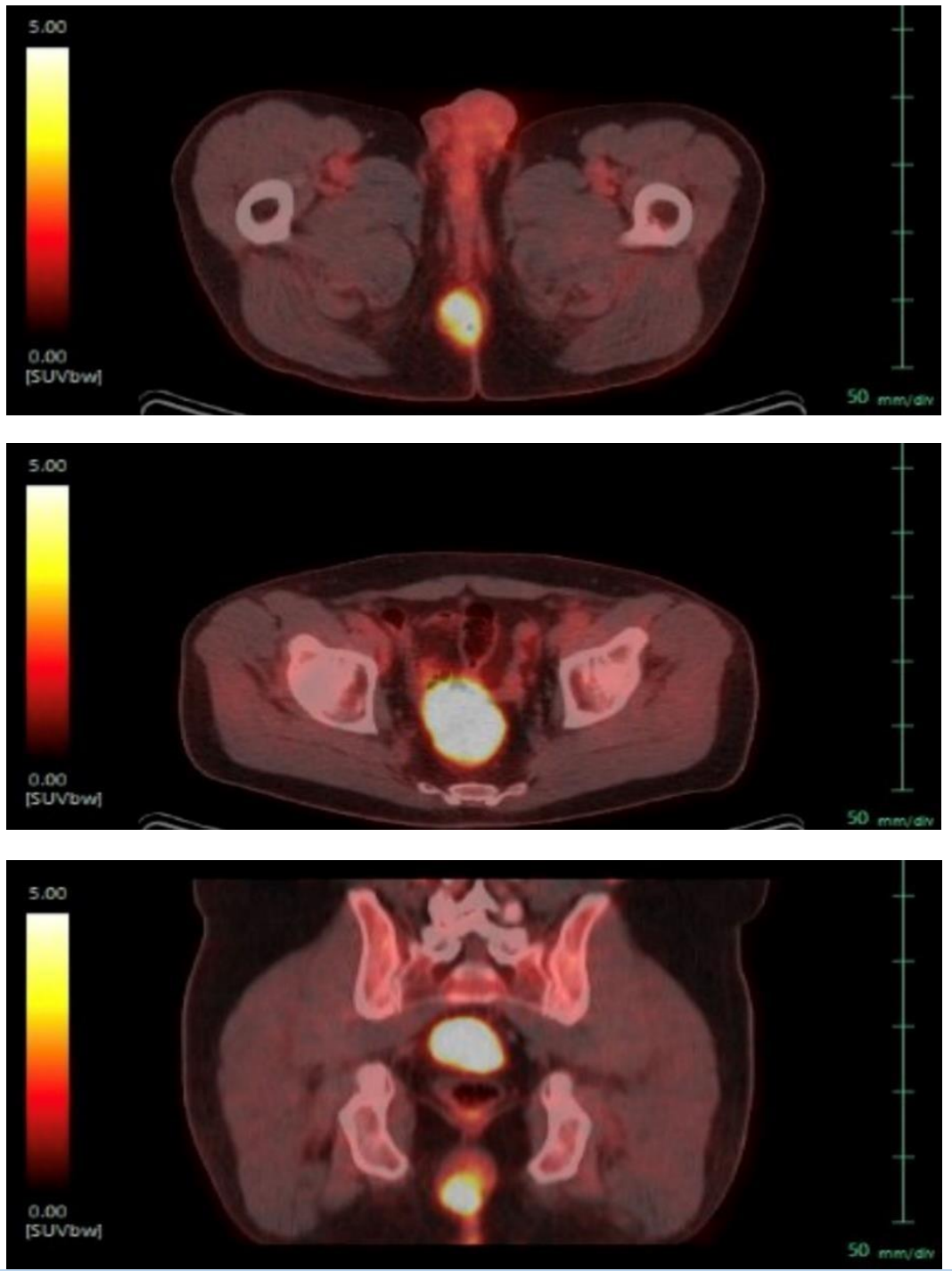

E. PET scans of the rectal lesion showing hot focus in the perirectal abscess 
The patient was taken in for a colonoscopy, and a mass higher up in the mid-rectal region was found and biopsied. Pathology results were identical to those of the previous rectal lesion, which is $(+)$ for moderately differentiated colonic adenocarcinoma, and an immunohistochemistry which was (+) CK20, (+) Cdx2 and (-) Ck7; the classic IHC staining pattern for colonic adenocarcinoma. The patient's case was discussed in the multimodal tumor board conference later that week, and he was started on a chemotherapy and radiation treatment for 8-12 weeks. Upon completion of this treatment, the plan is to then wait for 8 weeks, and then proceed with an abdomino-perineal resection (APR) procedure, followed by a systemic chemotherapy regimen.

\section{Discussion:}

There is a wide variability in the clinical presentation of cases of perianal mucinous adenocarcinomas. In the past, a 75-yr old female presented with an ulcerative growth and a 4-month history of difficulty defecating, which was diagnosed as $\mathrm{PMA}^{4}$. In the same report was another case of a 57-yr old man who presented with on and off bleeding per rectum for 6 months, leading up to a diagnosis of $\mathrm{PMA}^{4}$. Furthermore, a 60 -yr old man was diagnosed with PMA following a 45-yr history of long-standing perianal fistulas which were giving out a mucinous discharge, along with pain and induration in the region ${ }^{5}$. Another case in the literature has been that of a 56-yr old man whose PMA diagnosis was following 2-years of a recurrent fistula and complaints of painful defecation and blood mixed stools for about 6 months $^{1}$. In addition to the cases above, a study conducted by Anthony et al, exploring cases of PMA also stated that $75 \%$ of the patients had a history of chronic perirectal disease - which our patient did not have, thereby making his clinical presentation unique ${ }^{6}$. The presentation of our patient with a perirectal abscess and short history of burning sensation on sitting adds to the wide spectrum of PMA's clinical presentations and supports the fact that there is great variability in the ways in which PMA can present.

Past literature has also shown the presence of skip lesions in the context of anal carcinomas all the way up to the rectum, but they tend to be of the squamous type ${ }^{10}$. To the best of our knowledge, our case is the first where a perianal mucinous adenocarcinoma has presented with a skip lesion all the way up in the mid-rectal region. Additionally, the unique element of this case was that it presented without any long-standing defecation issues, long standing fistulas or recurrent abscesses, quite contrary to the ways in which some of the cases mentioned above have presented.

In terms of an early diagnosis, the difficulty arises in the slow and indolent growth of the PMA lesion which is hidden within the ischioanal fossa in many cases ${ }^{5}$. Literature has supported the use of CT imaging as the best diagnostic modality with respect to evaluating rectal lesions with a possible suspicion of malignancy because it allows us to detect tumor size and extension ${ }^{7}$. While MRI's can help elucidate tumor wall infiltration, and PET scans can help with a more holistic detection, at present, guidelines from the NCCN suggest an integrated PET/CT scan as the optimal tool for pretreatment staging evaluation for anal canal cancers ${ }^{9}$. In terms of management, the NCCN guidelines currently propose a continuous infusion of 5FU/mitomycin based radiation therapy (unless it's a small and well differentiated anal margin lesion), followed by an APR for patients with locoregional progressive disease following primary treatment ${ }^{8}$. Upon remission, a groin dissection for inguinal node metastasis or inguinal node dissection for regional recurrence in inguinal nodes can be performed ${ }^{8}$.

In conclusion, by taking into consideration the unique variants and atypical presentations of perianal mucinous adenocarcinomas, a better understanding towards different diagnostic and therapeutic approaches can be developed. It is important to present rare cases of PMA such as this one to multimodality speciality groups and tumor boards, so that the medical community can improve their understanding and appreciation for the numerous ways in which anal adenocarcinomas can present, and rectal lesions should be worked up. As a further step to our patient's case, investigations can be done to determine whether the perirectal abscess was a metastasis from the lesion or whether it was a second primary. This too can help enhance our knowledge and build a database on an even rare entity which we presented in this report - perianal mucinous adenocarcinomas with skip lesions.

\section{Reference}

1. Ryan, D., et al. (2019). Clinical features, staging and treatment of anal cancer.

2. Marti, L., et al. (2001). Perianal mucinous adenocarcinoma. A further reason for histological study of an anal fistula or anorectal abscess. J Chirug 72 (5): 573-7.

3. Lee, J., Kim, N. (2018). Essential Anatomy of the Anorectum for Colorectal Surgeons Focused on the Gross Anatomy and Histological Findings. J Ann Coloproctol. 34 (2): 59-71.

4. Kulkarni, M., et al. (2016). Adenocarcinoma of the anal canal: A report of two cases with review of literature. Indian J Pathol Microbiol; 59 (3): 404-6.

5. Ohta, R., et al. (2013). A Case of Perianal Mucinous Adenocarcinoma Arising from an Anorectal Fistula successfully resected after preoperative radiotherapy. Case Rep Gastroenterol May-Aug 7(2): 2019-223.

6. Schaffzin, D., et al. (2003). Perianal mucinous adenocarcinoma: unusual case presentations and review of the literature. J Am Surg; 69(2): 166-9.

7. Alvin, S., et al. (2006). Evaluation of Benign and Malignant Rectal Lesions with CT colonography and endoscopic correlation, $J$ Radiographics; 26(4).

8. Ajani, A., et al. (2016). NCCN Guidelines Version 1.2016 Anal Carcinoma Principles of Chemotherapy.

9. Glynne-Jones, R., et al. (2014). Anal cancer: ESMO ESSO ESTRO clinical practice guidelines for diagnosis, treatment and follow-up. J Radiother Oncol; 111(3): 330-9.

10. Durot, C., et al. (2017). Cancer of the Anal Canal: Diagnosis, Staging and Follow-up with MRI. Korean J Radiol; 18(6): 946956. 